\title{
In situ Transmission Electron Microscopy for Data-driven Modeling of Nanoparticle Evolution
}

James Horwath ${ }^{1}$, Peter Voorhees ${ }^{2}$ and Eric Stach $^{3}$

${ }^{1}$ University of Pennsylvania, United States, ${ }^{2}$ Northwestern Universtiy, United States, ${ }^{3}$ University of Pennsylvania, Philadelphia, Pennsylvania, United States

Supported metal nanoparticles are important for industrial catalysis. Their high surface-to-volume ratio produces highly active heterogeneous catalysts, however this high surface energy also provides a driving force for particle growth to stabilize the nanoparticles. Though several mechanisms are expected to contribute to particle growth, and previous experiments have verified their existence in real systems, our understanding of the evolution of supported nanoparticles is based on mean-field theories which cannot describe the behavior of real, non-ideal systems. In situ Transmission Electron Microscopy (TEM), paired with high-framerate direct electron detection cameras, is particularly well suited to studying dynamic processes as it allows us to observe changes with high resolution in both space and time.

We performed high temperature in situ heating experiments on a model system of supported catalytic nanoparticles. Aided by advances in unsupervised deep learning meant to optimize the speed and accuracy of automated image segmentation [1], a time series of hundreds of experimental images was processed to yield a large volume of data describing the evolution of individual nanoparticles as a function of time at elevated temperature. This approach to the extraction and quantification of data from experimental images allowed us to obtain both standard analyses of the bulk system, for example characterization of the particle size distribution as a function of time, and detailed examinations of the behavior of individual particles, such as directly tracking short-range interactions between particles (Figure 1).

After observing a global decrease in particle size through the course of the experiment and determining that this loss of mass could not be described by evaporation alone, an analytical model was developed from classical thermodynamics and kinetics describing the change in particle size due to diffusion along the support surface and evaporation from the particle surface. Utilizing the extensive database built from the experiment, individual particle trajectories were fit to this model so that physical parameters could be extracted with statistical certainty. Comparing the extracted parameter values to data found in the literature, the system could be directly characterized in terms of parameters which cannot be easily measured from standard TEM experiments, such as the particle interaction distance and the adatom concentration at the interface between a particle and the support. With this method we can gauge the relative impact of each mechanism on the overall coarsening rate and understand how the experimental conditions influence the behavior of the particles. Though several sources discuss the impact of surface free energy on the size-dependent evolution of nanoparticles [2,3], we show that in our experiment, where continual pumping of the experiment chamber causes mass not to be conserved, the elevated vapor pressure of the small metal particles (described by the Gibbs-Thomson and Kelvin Effects) dominates the energy penalty for increasing the surface-to-volume ratio, thus causing nanoparticles to shrink until they disappear. Similarly, we consider the interactions between neighboring nanoparticles in terms of their arrangement and separation distance on the support to show that the screening length derived from our model is closely related to the distance over which the particle array is ordered. 
Overall, a novel approach to experimental data analysis, paired with a model based on fundamental physics, provides a clearer understanding of the interplay of coarsening mechanisms which cause the degradation of supported catalyst.
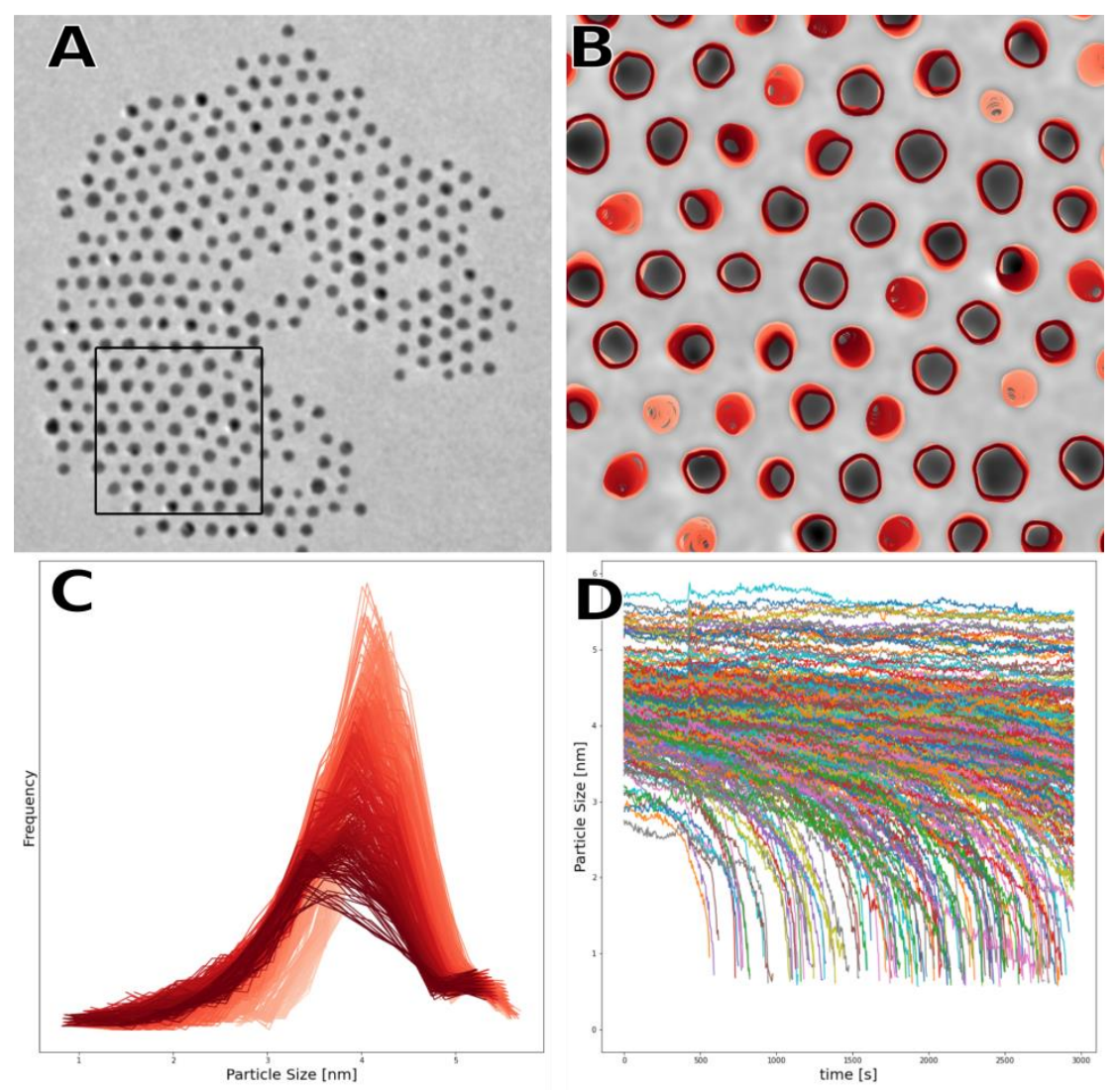

Figure 1. Demonstration of in situ TEM data analysis. A.) shows the ordered array of self-assembled nanoparticles. The box in the bottom left shows the region magnified in B.), where particle outlines are shown as a function of time (the shade of red darkens as time increases). The same color scale is used in C.) where traditional particle size distribution analysis shows decreasing particle size and a broadening of the distribution. D.) shows the detailed analysis of individual particles. Intersecting trajectories shows that all particles do not behave the same.

\section{References}

1. Horwath, D. Zakharov, R. Megret, E. Stach, "Understanding important features of deep learning models for segmentation of high-resolution transmission electron microscopy images", npj Computational Materials 6108 (2020).

2. Blackman, N. Lisgarten, L. Skinner, "Surface energy and evaporation rate of spherical particles of radii less than $500 \AA$ ", Nature 217 1254-1246 (1968).

3. Nanda, F. Kruis, H. Fassan, "Evaporation of Free PbS Nanoparticles: Evidence of the Kelvin Effect," Physical Review Letters 89256103 (2002).

4. P.H and E.A.S acknowledge support through the National Science Foundation, Division of Materials Research, Metals and Metallic Nanostructures Program under Grant 1809398. This research used resources of the Center for Functional Nanomaterials, which is a U.S. DOE Office of Science Facility, at Brookhaven National Laboratory under Contract No. DE-SC00127044 